VIEWPOINT

Paulus T. V. M. de Jong, MD, PhD

Department of Ophthalmogenetics, Netherlands Institute of Neurosciences, Royal Netherlands Academy of Arts and Sciences, Amsterdam, the Netherlands, Department of Ophthalmology, Academic Medical Center, Amsterdam, the Netherlands, and Department of Ophthalmology, Leiden University Medical Center, Leiden, the Netherlands.

\section{Jie Jin Wang, MMed,}

PhD

Centre for Vision

Research, Department of Ophthalmology and the Westmead Millennium Institute, University of Sydney, Sydney, Australia, and Centre for Eye Research Australia, University of Melbourne, Royal Victorian Eye and Ear Hospital, Melbourne, Australia.

Astrid E. Fletcher, PhD Faculty of Epidemiology and Population Health, London School of Hygiene and Tropical Medicine, London, United Kingdom.

Corresponding Author: Jie Jin Wang, MMed, PhD, Centre for Vision Research, Westmead Millennium Institute, University of Sydney C24, Westmead Hospital, NSW 2145, Australia (jiejin.wang @sydney.edu.au).

\title{
Aspirin Use and Aging Macula Disorder
}

For nearly half a century, associations between aspirin use and aging macula disorder (also termed agerelated macular degeneration [both $\mathrm{AMD}$ ]) have been described, predominantly from case reports and small clinical studies. In the last year, the publication of more robust evidence from 3 population-based studies has reawakened interest in this question. ${ }^{1-3}$

One cross-sectional ${ }^{1}$ and 2 prospective ${ }^{2,3}$ studies found an increased risk of AMD with regular aspirin use. In the cross-sectional European Eye Study, the odds ratio for neovascular AMD (nAMD) rose with more frequent aspirin use, and for daily aspirin use, the odds ratio was 2.22 (95\% Cl, 1.61-3.05). ${ }^{1}$ In the 20-year follow-up of the Beaver Dam Eye Study, the risk for incident nAMD with regular use of aspirin, as reported 10 years previously, was 2.20 (95\% Cl, 1.20-4.15). ${ }^{2}$ In the 15 -year follow-up of the Blue Mountains Eye Study, the cumulative risk of regular use of aspirin for incident nAMD was $2.46(95 \% \mathrm{Cl}, 1.25-4.83) .{ }^{3}$ None of the studies found an association with geographic atrophy, the dry form of late AMD. The European Eye Study also found an association with early AMD. ${ }^{1}$

Given the estimated $19 \%$ of the US population using aspirin regularly, ${ }^{2}$ these results warrant closer scrutiny. The 3 studies $^{1-3}$ were observational studies and limitations in study design need to be considered. Confounding by indication or "indication bias," a major concern when assessing drug-related risks in nonrandomized studies, was examined in all 3 studies. The associations of aspirin with AMD were not explained by cardiovascular disease (CVD) history, ${ }^{1-3}$ the main reason for regular aspirin use. Nor was there any evidence that the effects of aspirin differed in those with CVD..$^{1-3}$ In the Blue Mountains EyeStudy, the odds ratios for aspirin use were higher in those with a history of CVD but the number of cases was small and the $P$ value for effect modification testing was .30. In the other studies, there was no significant effect modification and no difference in the odds ratios when stratified by CVD history. ${ }^{1,2}$

There was no association with other types of painrelieving medications and AMD. ${ }^{3}$ Although there was limited information on other conditions for which aspirin might be taken, no association or effect modification was found with a history of arthritis in the study that investigated this. ${ }^{2}$ Aspirin use was self-reported in all studies and in 2 studies ${ }^{2,3}$ corroborated with information from prescriptions or actual medications the participants were using. There were no dosage data of aspirin, although 1 study estimated a daily dose in milligrams and found no significant relationship between dose and early or late AMD. ${ }^{2}$ Respondent errors in reporting aspirin use dose and frequency are expected, especially over a longterm follow-up period. These errors are likely to dilute the observed associations. The number of nAMD cases was low: of 10212 participants in the 3 studies, $^{1-3} 208$
(2\%) were nAMD cases, 108 prevalent and 100 incident, leading to low power in subgroup analyses.

How do these results fit with other studies? Older publications (reviewed in the European Eye Study article ${ }^{1}$ ) often mixed anticoagulant and antiplatelet aggregation drugs, were not prospective, and mostly had no controls. Two randomized clinical trials, the Physicians' Health Study I ${ }^{4}$ and the Women's Health Study, ${ }^{5}$ found no effect of aspirin on AMD over the durations of 5 and 10 years. Age-related macular degeneration was self-reported and confirmed by medical records. Despite the large sample sizes of the 2 trials ( 21000 and 40000 , respectively), there were very few cases of visually impairing late AMD (12 nAMD and 4 geographic atrophy $)^{4}$ and 55 cases of combined nAMD and geographic atrophy, ${ }^{5}$ and AMD incidence was at least 10 times lower than in large population-based studies. 2,3,6 In the Women's Health Study, the hazard ratio for all late AMD was $0.90(95 \% \mathrm{Cl}, 0.53-1.52)^{5}$; no equivalent estimate was provided for the Physicians' Health Study I. ${ }^{4}$ Neither trial presented the results by subtype of late AMD. Low power and lack of results on nAMD make it difficult to assess the contribution of these 2 trials to the question of whether regular aspirin use increases the risk of nAMD.

The recent 3 studies $^{1-3}$ with validated AMD diagnosis based on fundus image grading showed similar associations, pointing to some consistency and specificity of the association of regular aspirin use with nAMD but not with geographic atrophy. The prospective studies imply that exposure to aspirin preceded the occurrence of $A M D$ (temporality); the longer the follow-up, the stronger the association magnitude was in the Blue Mountains EyeStudy; while in the Beaver Dam EyeStudy, use of aspirin, reported 10 years earlier but not 5 years earlier, increased the risk of nAMD. There was some evidence suggesting a dose-response pattern in the association, with increasing odds ratios for increasing frequency of aspirin use in the 2 studies ${ }^{1,3}$ that assessed this. There are indications for biological plausibility such as inhibiting prostaglandin-endoperoxide synthetase 1 and 2 by aspirin, inhibition of transporting protons, and formation of nitric oxygen radicals. ${ }^{1}$ We therefore conclude that the evidence is sufficient to justify caution about this potential long-term risk of nAMD associated with regular use of aspirin.

The benefit of aspirin for secondary prevention of CVD is well established. A meta-analysis of low-dose aspirin trials found a reduction in the annual recurrence of serious vascular events ( $6.7 \%$ in those randomized to aspirin compared with $8.2 \%$ in controls). ${ }^{7}$ For those with no history of CVD (primary prevention), the benefit was substantially less ( $0.51 \%$ annual incidence with aspirin compared with $0.57 \%$ in controls), reflecting a much lower absolute risk reduction in those with no history of 
CVD. Conversely, in the primary prevention group, aspirin was associated with an increase in adverse events. There was no benefit in ischemic stroke, an increase in hemorrhagic stroke, and an increase in gastrointestinal and extracranial bleeding.

What should ophthalmologists advise their patients in light of these findings? The small risk of regular aspirin use in nAMD, a serious but uncommon condition, needs to be balanced against the benefit of aspirin together with other varying risks, according to the individual patient's conditions and CVD risk factors, including age. It would seem prudent that ophthalmologists advise patients with known AMD in at least 1 eye and no history of CVD to consult their family physicians or cardiologists as to whether regular aspirin use as primary CVD prevention is warranted. Recommendations for aspirin use might be better individually tailored in the future, with improved prediction models for AMD, based on environmental and genetic profiles.

\section{ARTICLE INFORMATION}

Published Online: October 31, 2013. doi:10.1001/jamaophthalmol.2013.5193.

Conflict of Interest Disclosures: None reported.

REFERENCES

1. de Jong PTVM, Chakravarthy U, Rahu M, et al. Associations between aspirin use and aging macula disorder: the European Eye Study. Ophthalmology. 2012;119(1):112-118.

2. Klein BEK, Howard KP, Gangnon RE, Dreyer JO, Lee KE, Klein R. Long-term use of aspirin and age-related macular degeneration. JAMA. 2012;308(23):2469-2478.
3. Liew G, Mitchell P, Wong TY, Rochtchina E, Wang

$\mathrm{JJ}$. The association of aspirin use with age-related macular degeneration. JAMA Intern Med. 2013;173(4):258-264.

4. Christen WG, Glynn RJ, Ajani UA, et al. Age-related maculopathy in a randomized trial of low-dose aspirin among US physicians. Arch Ophthalmol. 2001:119(8):1143-1149.

5. Christen WG, Glynn RJ, Chew EY, Buring JE. Low-dose aspirin and medical record-confirmed age-related macular degeneration in a randomized trial of women. Ophthalmology. 2009;116(12) 2386-2392.
6. van Leeuwen $R$, Klaver CC, Vingerling JR, Hofman A, de Jong PTVM. The risk and natural course of age-related maculopathy: follow-up at $61 / 2$ years in the Rotterdam study. Arch Ophthalmol. 2003;121(4):519-526.

7. Baigent C, Blackwell L, Collins R, et al; Antithrombotic Trialists' (ATT) Collaboration. Aspirin in the primary and secondary prevention of vascular disease: collaborative meta-analysis of individual participant data from randomised trials. Lancet. 2009;373(9678):1849-1860. 